Cite this: Phys. Chem. Chem. Phys., 2013, 15, 19019

Received 8th August 2013,

Accepted 20th September 2013

DOI: $10.1039 /$ c3cp53376j

www.rsc.org/pccp

\title{
Role of preferential weak hybridization between the surface-state of a metal and the oxygen atom in the chemical adsorption mechanism
}

\author{
Yong Su Kim,$\dagger^{\mathrm{ab}}$ Sang Ho Jeon,$\dagger^{\mathrm{c}}$ Aaron Bostwick, ${ }^{a}$ Eli Rotenberg, ${ }^{a}$ Philip N. Ross, ${ }^{d}$ \\ Andrew L. Walter, ${ }^{\text {ae }}$ Young Jun Chang, ${ }^{\text {af }}$ Vojislav R. Stamenkovic, ${ }^{9}$ \\ Nenad M. Markovic, ${ }^{9}$ Tae Won Noh, ${ }^{\text {b Seungwu Han }}{ }^{\star c}$ and Bongjin Simon Mun ${ }^{\star h}$
}

\begin{abstract}
We report on the chemical adsorption mechanism of atomic oxygen on the $\mathrm{Pt}(111)$ surface using angleresolved-photoemission spectroscopy (ARPES) and density functional calculations. The detailed band structure of Pt(111) from ARPES reveals that most of the bands near the Fermi level are surface-states. By comparing band maps of Pt and O/Pt, we identify that $d_{x z}\left(d_{y z}\right)$ and $d_{z^{2}}$ orbitals are strongly correlated in the surface-states around the symmetry point $M$ and $K$, respectively. Additionally, we demonstrate that the s- or p-orbital of oxygen atoms hybridizes preferentially with the $d_{x z}\left(d_{y z}\right)$ orbital near the $M$ symmetry point. This weak hybridization occurs with minimal charge transfer.
\end{abstract}

\section{Introduction}

Surface oxidation, i.e. the chemical adsorption (chemisorption) of oxygen (O), on a Pt-based catalyst is a key process for industrial catalytic processes, such as $\mathrm{CO}$ and $\mathrm{NO}_{x}$ oxidation and O-reduction reactions. ${ }^{1-10}$ Over the years, the nature of the adsorbed $\mathrm{O}$ on the Pt surface, e.g., molecular $\mathrm{O}$ and chemisorbed atomic $\mathrm{O}$, has been extensively investigated. At low temperatures, $\mathrm{O}$ molecules initially adhere to the Pt surface by physisorption. Then, as temperature increases, they form two distinct intermediate states: a superoxide $\left(\mathrm{O}_{2}{ }^{-}\right)$and a peroxide $\left(\mathrm{O}_{2}{ }^{2-}\right)$ state. These intermediate states further dissociate into atomic $\mathrm{O}$ at elevated temperatures $(T>150 \mathrm{~K})$, introducing stable chemisorbed $\mathrm{O}$ atoms into the face-centered cubic (fcc) hollow site of Pt(111). ${ }^{11-14}$

\footnotetext{
${ }^{a}$ Advanced Light Source, Lawrence Berkeley National Laboratory, Berkeley, California 94720, USA

${ }^{b}$ CFI-CES, IBS and Department of Physics \& Astronomy, Seoul National University, Seoul 151-747, Korea

${ }^{c}$ Department of Materials Science and Engineering, Seoul National University, Seoul 151-744, Korea. E-mail: hansw@snu.ac.kr

${ }^{d}$ Materials and Molecular Research Division, Lawrence Berkeley National Laboratory, Berkeley, California 94720, USA

${ }^{e}$ Department of Molecular Physics, Fritz-Haber-Institut der Max-Planck-Gesellschaft, 14195 Berlin, Germany

${ }^{f}$ Department of Physics, University of Seoul, Seoul 130-743, Korea

${ }^{g}$ Materials Science Division, Argonne National Laboratory, Argonne, Illinois 60439, USA

${ }^{h}$ Department of Physics \& Photon Science, ERTL Center,

Gwangju Institute of Science and Technology, Gwangju 500-712, Korea.

E-mail: bsmun@gist.ac.kr

$\dagger$ These authors contributed equally to this work.
}

In recent years, the intermediate states or precursor states, which are initially formed in the chemisorption processes of oxygen on the Pt surface, have received much attention due to their critical role in determining the kinetics of dissociative chemisorption. ${ }^{13,15}$ Consequently, for the past decades, various physical-chemical properties of the intermediate states such as the geometrical configuration, ${ }^{16-18}$ charge assignment, ${ }^{8}$ and the electronic properties ${ }^{14}$ have been intensively studied.

On the other hand, the formation of the chemisorbed state of atomic oxygen, i.e., the final step in the chemisorption of $\mathrm{O}$ on the Pt surface, plays an important role in the catalytic activity of Pt-based catalysts. The chemical bonding interaction between atomic oxygen and the Pt surface is a determining factor for catalytic activity. Up to now, countless theoretical and experimental studies have been devoted to identifying the electronic origin of the surface reactivity. Notably, with the distribution properties of d-electrons near the Fermi level, the correlations between the surface reactions and electronic structures are estimated, i.e., the d-band model. ${ }^{19}$ According to the d-band model the precise bonding mechanism and critical physical parameters for surface reactions can be retrieved from the analysis of surface electronic structure. In the study of chemisorbed atomic oxygen on the Pt surface, major focus, to date, has been on the adsorption properties of atomic $\mathrm{O}$ on the Pt surface, such as the adsorption site, ${ }^{20}$ adsorbate growth, ${ }^{21}$ and adsorption energy. ${ }^{22}$ Nevertheless, there have been few experimental investigations on the surface electronic structure of $\mathrm{Pt}$ on which the chemisorbed $\mathrm{O}$ resides. In fact, even for pure Pt, there is a lack of knowledge about the detailed surface electronic structure. 
Here, with high performance angle-resolved photoemission spectroscopy (ARPES), we report the direct visualization of the detailed surface electronic band structure of $\mathrm{Pt}(111)$ and $\mathrm{O} / \mathrm{Pt}(111)$, which leads to the understanding of the fundamental bonding mechanism between chemically-adsorbed atomic $\mathrm{O}$ and the $\mathrm{Pt}$ surface. ARPES is a surface-sensitive probe that can resolve both energy and momentum space. Therefore, this technique provides unique access to the valence band structure and surface electronic structure of materials. Supported by density functional theory (DFT) calculations, the band structures measured by ARPES can disclose the details of orbital structures and the degree of hybridization (and charge transfer) in surface electronic structures. According to our experimental results, we found that only one type of surface-state in the valence band substantially participates in the preferential weakhybridization during the chemical adsorption process. Specifically, a surface state near the M symmetry point with the $\mathrm{d}_{x z}\left(\mathrm{~d}_{y z}\right)$ orbital initiates weak hybridization with the s- or p-orbital of the chemisorbed atomic $\mathrm{O}$, and shows minimal charge transfer between the Pt surface and $O$. This detailed knowledge of the surface electronic structure of Pt-O bonding, which to our knowledge has not been clearly identified previously, provides a fundamental basis for resolving the chemical adsorption mechanism of $\mathrm{O}$ atoms on all Pt-based catalytic surfaces.

\section{Results and discussion}

\subsection{Preparation of chemisorbed atomic oxygen on a clean Pt(111) surface}

A clean Pt $(111)$ surface, showing a well-ordered $(1 \times 1)$ structure, was prepared by cycles of sputtering and annealing. No carbon or $\mathrm{O}$ contamination was detected on the prepared surface by $\mathrm{X}$-ray photoemission spectroscopy. On the clean surface, $\mathrm{O}$ was dosed at $1 \times 10^{-7}$ Torr for $5 \mathrm{~min}$ at $30 \mathrm{~K}^{23}$ After dosing with $\mathrm{O}$, the Pt surface was gradually heated to $T>150 \mathrm{~K}$ so as to ensure that chemisorbed $\mathrm{O}$ is mainly composed of atomic oxygen. The $(2 \times 1)$ structure was confirmed by LEED experiments. ${ }^{23}$ The band structures were measured at $\sim 40 \mathrm{~K}$. High-resolution ARPES was conducted at beamline 7.0.1 of the Advanced Light Source.

\subsection{High-resolution angle resolved photoemission spectroscopy}

At first, in order to find the symmetry point where the bonding between $\mathrm{O}$ and the Pt surface occurred, the Fermi surfaces, $E_{\mathrm{F}}$, of clean $\mathrm{Pt}(111)$ and $\mathrm{O} / \mathrm{Pt}(111)$ were mapped as shown in Fig. 1a and b, respectively. A comparison of the Fermi surfaces of the clean Pt and $\mathrm{O} / \mathrm{Pt}$ surfaces shows small changes near the $\mathrm{M}$ point, although these changes are not substantial. Previous studies showed that the dissociative adsorption of intermediate states, i.e., $\mathrm{O}_{2}{ }^{-}$and $\mathrm{O}_{2}{ }^{2-}$, onto chemisorbed atomic $\mathrm{O}$ is followed by hybridization between the surface $\mathrm{Pt}$ atom and $\mathrm{O}$ adsorbate; this process is accompanied by charge transfer from the Pt atom to atomic $\mathrm{O},{ }^{14,24}$ In the case of strong hybridization between a metal surface and an adsorbate, such as graphene ${ }^{25}$ and $O{ }^{26}$ observable features in the band structure can be monitored by observing the charge transfer process. However, in Fig. 1b, the Fermi surface of Pt with chemisorbed atomic $\mathrm{O}$ does not show any indication of strong hybridization. The Fermi surface change associated with charge transfer will be further discussed below.

Details of the band structure changes due to hybridization can be found in the band cut along the $Г \mathrm{KM}$ direction of the (a)

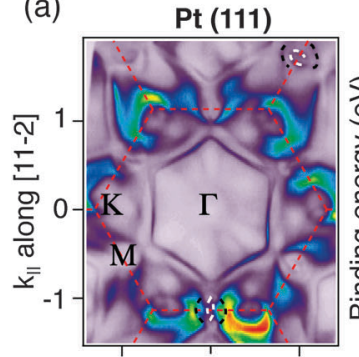

(b)

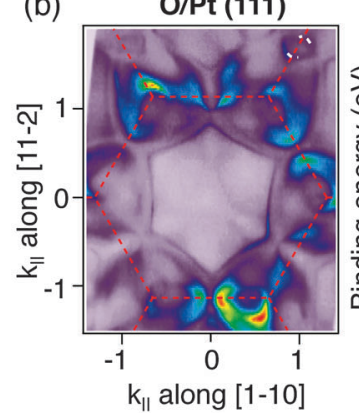

(c)

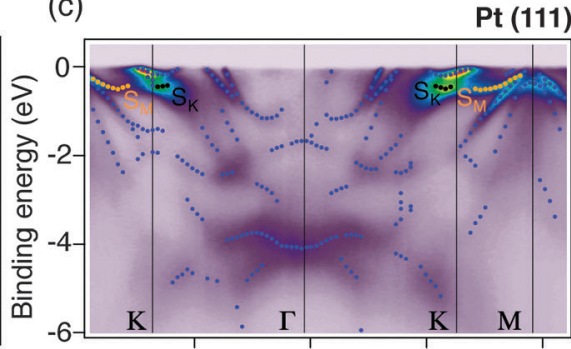

(d)

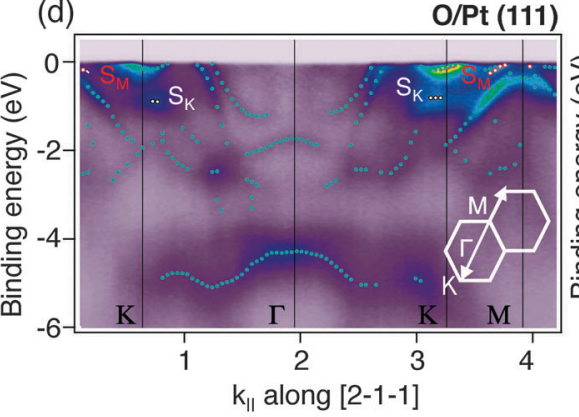

(e)

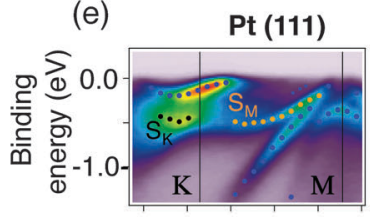

(f)
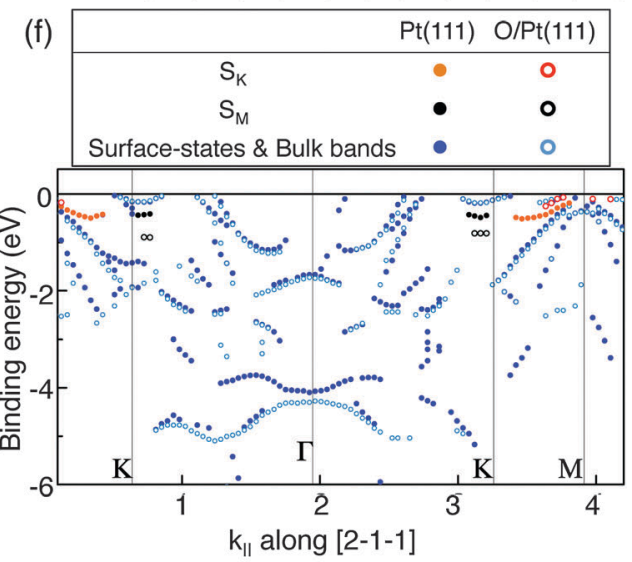

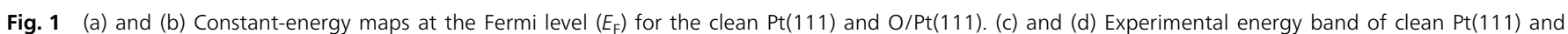

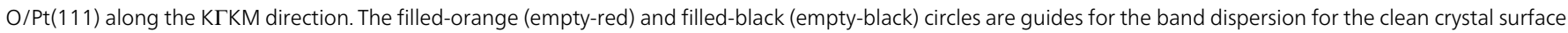

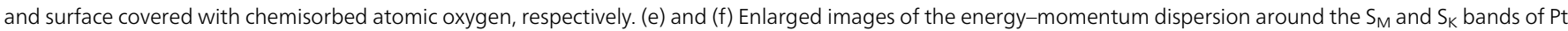

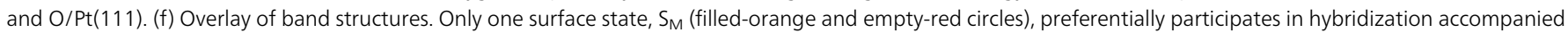
by slight charge-transfer from $\mathrm{Pt}$ to $\mathrm{O}$ atom. 
Brillouin zone for clean Pt and O/Pt surfaces (Fig. 1c and d, respectively). The dotted lines are guides for the observed band dispersion. For a better comparison, the band structures of clean $\mathrm{Pt}$ and $\mathrm{O} / \mathrm{Pt}$ are plotted together in Fig. 1e.

To analyze the bonding interaction between the Pt surface and chemisorbed atomic $\mathrm{O}$, we first need to refer to the electronic band structure of clean Pt(111). Interestingly, most of the bands near the Fermi level are surface states and resonances (hereafter, referred to as surface-states) associated with clean Pt (Fig. 1c). To distinguish the bulk band and surface-states in the band structure shown in Fig. 1c, we utilized two experimental methods. First, to identify the surface state, we performed band mapping for a clean $\mathrm{Pt}(111)$ and one dosed with hydrogen, $\mathrm{H} / \mathrm{Pt}(111)$, and observed a change in the band structure, due to chemical bonding between the hydrogen atoms and the surface (not shown here). Then, as a more convincing way of identifying the surface-states, we probed the band structure along the $k_{\perp}$ (surface normal) direction. Surface-states are strongly localized in the topmost surface layer. Therefore, they are sensitive to chemical bonding between the adsorbate and the surface atom and, more critically, they show little dispersion along the surface normal direction in momentum space.

Fig. 2 shows the energy-momentum dispersion relationship along the perpendicular momentum direction, $k_{\perp}$, to the $\operatorname{Pt}(111)$ surface. We chose the same ARPES map as in Fig. 1c. Then, as shown in Fig. 2a, we collected the ARPES map along $k_{\perp}$ (surface normal) by scanning the photon energy from 100 to $160 \mathrm{eV}$. Fig. 2b-d show the constant-energy cuts of the spectral function at different binding energies, i.e., $E_{0}, E_{1}$, and $E_{2}$. The blue

(a)

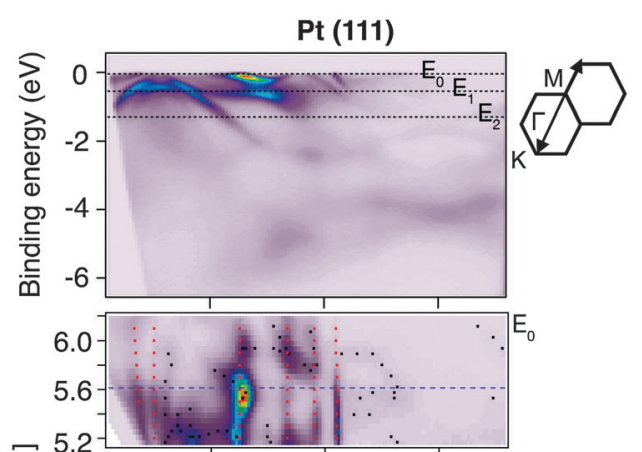

(c)

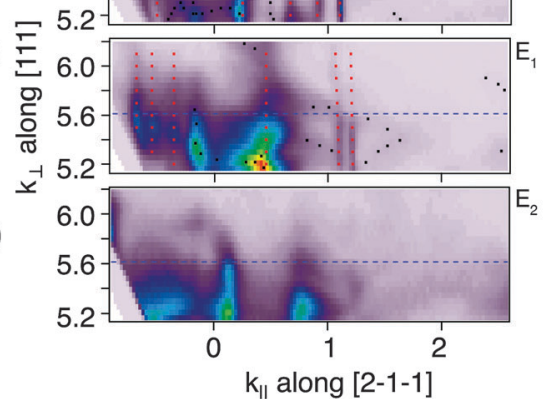

Fig. 2 (a) Experimental energy band along the high symmetry direction, $k_{\|}$, for the clean Pt(111) surface. (b)-(d) Constant-energy cuts of the spectral function $\left(k_{\perp}\right.$ dispersion) at different binding energies, i.e., $E_{0}, E_{1}$, and $E_{2}$ in the ARPES band shown in (a). The blue dashed-line indicates the photon energy of $120 \mathrm{eV}$, i.e., energymomentum dispersion cut shown in (a). The black and red dots are guides for the band dispersion for some bulk states and surface-states, respectively. dashed lines indicate the photon energy of $120 \mathrm{eV}$, which corresponds to the energy-momentum dispersion cut shown in Fig. 2a. The straight dispersions in the constant-energy cuts of the spectral function (Fig. 2b-d) correspond to surface-states. From this result, many bands near the Fermi level of clean Pt are localized along the $k_{\perp}$ direction, an identifying feature of surface-states.

Another important feature in the ARPES band maps in Fig. 1c and $d$ is that only two surface-states, $S_{M}$ and $S_{K}$, distinguished by filled-orange (empty-red) and filled-black (empty-black) circles, show remarkable changes of band dispersion before and after $\mathrm{O}$ dosing. Considering hybridization accompanied by charge-transfer from $\mathrm{Pt}$ to $\mathrm{O}$ atom, we determined that only the surface state $\mathrm{S}_{\mathrm{M}}$ preferentially contributed to charge transfer from $\mathrm{Pt}$ to the $\mathrm{O}$ atoms during hybridization, because $S_{M}$ was the only $d$ band that shifted closer to the Fermi level. The change in the Fermi surface near the $M$ point, caused by the up-shifting of $S_{M}$, was not significant enough to estimate the single negative state of the adsorbed atomic $\mathrm{O}$ induced by charge transfer. This result indicates that the charge transfer from $\mathrm{Pt}$ to the $\mathrm{O}$ atom is very small and the $\mathrm{O}$ atom undergoes preferential weak-hybridization on the $\operatorname{Pt}(111)$ surface. On the other hand, surface-state $S_{K}$ around the $K$ point shifts down after $\mathrm{O}$ dosing. However, in contrast to $S_{M}$, the $S_{K}$ state does not participate in the chemical bonding interaction with the chemisorbed atomic $\mathrm{O}$. This point will be discussed further in detail.

\subsection{Density functional theory calculations}

We performed first-principles calculations based on DFT on the $\mathrm{Pt}(111)$ and $\mathrm{O} / \mathrm{Pt}(111)$ surfaces to better understand the detailed characteristics and roles of the surface states, $\mathrm{S}_{\mathrm{M}}$ and $\mathrm{S}_{\mathrm{K}}$, in $\mathrm{O}$ chemical bonding. The projector augmented-wave potentials ${ }^{27}$ and generalized gradient approximation, ${ }^{28}$ as implemented in the VASP code, ${ }^{29}$ were adopted. The cut-off energy for the planewave basis is set to $400 \mathrm{eV}$. The Pt(111) surface is modeled by a slab consisting of 15 layers; the 7 layers in the middle were assigned to the bulk lattice parameter of $3.86 \AA$, while the other layers were fully relaxed until the atomic forces were reduced to within $0.03 \mathrm{eV} \AA^{-1}$. The energy and band-structure calculations were carried out for the $\mathrm{Pt}(111)$ and $\mathrm{O}($ fcc-hollow site)/Pt(111) geometries. The oxygen atom in $\mathrm{O} / \mathrm{Pt}(111)$ covers the fcc hollow site, the most stable site on the Pt(111) surface. ${ }^{30}$

Fig. 3a and $\mathrm{b}$ show the computed band structures of $\mathrm{Pt}(111)$ and $\mathrm{O} / \mathrm{Pt}(111)$, marked with dots. The ARPES data are also overlaid with the results of Fig. $3 \mathrm{a}$ and $\mathrm{b}$ for a close comparison. The experimental energy dispersions were well reproduced by the calculations. In Fig. $3 \mathrm{c}$ and $\mathrm{d}$, the band structures are colorcoded in blue and red according to the relative weights on $\mathrm{d}_{x z}$ (or equivalently $\mathrm{d}_{y z}$ ) and $\mathrm{d}_{z^{2}}$ of the surface Pt atoms, respectively. From Fig. 3, it is seen that the surface-states $S_{M}$ and $S_{K}$ have a mixed orbital character of $\mathrm{d}_{x z}\left(\mathrm{~d}_{y z}\right)$ and $\mathrm{d}_{z^{2}}$. However, it should be noted that weak hybridization between atomic $\mathrm{O}$ and the surface Pt atom produces clearly the orbital character of each band, that is, $\mathrm{d}_{x z}\left(\mathrm{~d}_{y z}\right)$ and $\mathrm{d}_{z^{2}}$ are dominant on $\mathrm{S}_{\mathrm{M}}$ and $\mathrm{S}_{\mathrm{K}}$, respectively. This indicates that the preferential hybridization between $\mathrm{s}$ or $\mathrm{p}$ orbitals of the $\mathrm{O}$ atom and the $t_{2 g}$ orbital of $\mathrm{S}_{\mathrm{M}}$ 

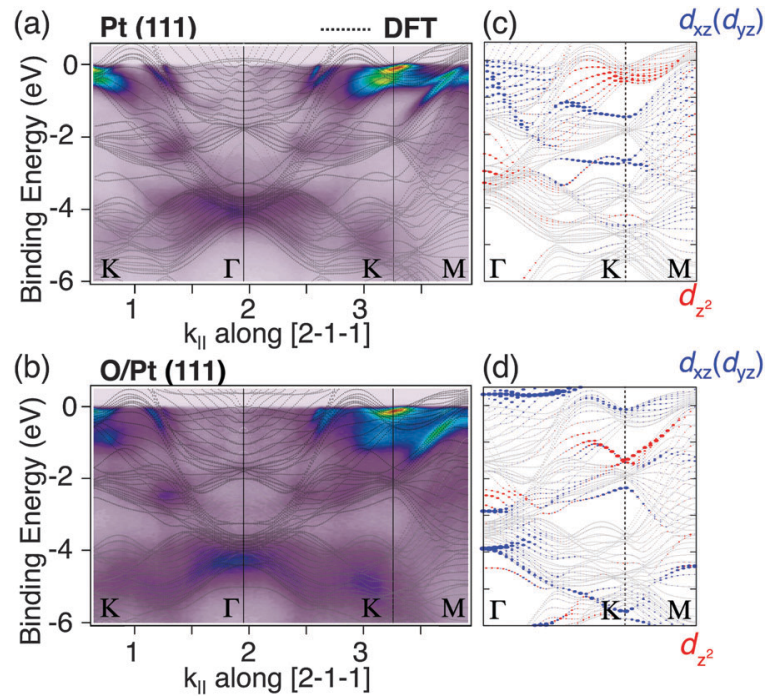

Fig. 3 (a) and (b) Experimental and calculated (dotted-line) band structure of $\mathrm{Pt}(111)$ and O/Pt(111) along the symmetric direction. (c) and (d) Projected wave function characters of the surface Pt layer. The orbital characters are marked as circles with radii corresponding to the projected weights. The surface-state near the M point, $S_{M}$, which is the only surface state involved in preferential hybridization accompanied by charge-transfer from Pt to the $\mathrm{O}$ atom shows strong $\mathrm{d}_{x z}\left(\mathrm{~d}_{y z}\right)$ character.

splits the d-orbital manifold around the symmetric $\mathrm{M}$ and $\mathrm{K}$ points. To be specific, among the five $d$ orbitals, $d_{x z}\left(d_{y z}\right)$ maximizes the overlap with the s or $\mathrm{p}$ orbitals of the $\mathrm{O}$ atom; this is consistent with the above analysis following Fig. 1, which indicates that the $S_{M}$ state is responsible for the major chemical interaction, i.e., the hybridization accompanied by charge transfer from $\mathrm{Pt}$ to $\mathrm{O}$, as indicated by the up-shift in the band structure.

Fig. 3 also shows that the down-shifted $\mathrm{S}_{\mathrm{K}}$ states at the $K$ point are mainly characterized by $\mathrm{d}_{z^{2}}$ orbitals. In Fig. $3 \mathrm{c}$ and $\mathrm{d}$, we identified that $\mathrm{d}_{x z}\left(\mathrm{~d}_{y z}\right)$ and $\mathrm{d}_{z^{2}}$ orbitals are strongly correlated in the surface-states $S_{M}$ and $S_{K}$. Therefore, when the s or p orbitals of $\mathrm{O}$ preferentially hybridize with the $\mathrm{d}_{x z}\left(\mathrm{~d}_{y z}\right)$ orbital around the $\mathrm{M}$ point, distortion is induced on the given orbital, resulting in a down-shift of $S_{K}$ in the surface layer.

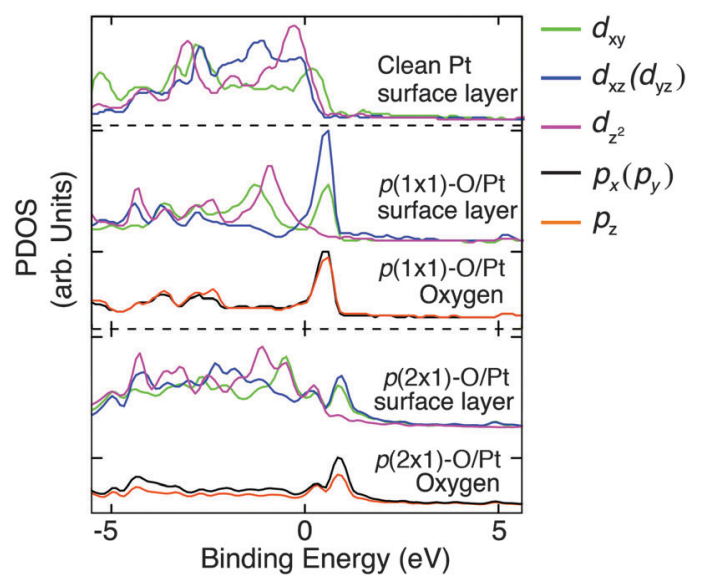

Fig. 4 Partial density of states (PDOS) for $d_{x z}\left(d_{y z}\right)$ and $d_{z^{2}}$ orbitals in the surface $\mathrm{Pt}$ layer of $\mathrm{Pt}(111)$ and $\mathrm{O} / \mathrm{Pt}(111)$ for different O coverage: $p(1 \times 1)$ and $p(2 \times 1)$ O/Pt(111).
To clearly illustrate the characteristics of d-orbitals in preferential hybridization, we calculated the partial density of states (PDOS) of the present system. Fig. 4 shows the PDOS for Pt(111) and $\mathrm{O} / \mathrm{Pt}(111)$ surfaces resolved over different orbitals in the surface layer and oxygen atoms. For comparison, results for $p(1 \times 1)-\mathrm{O} / \mathrm{Pt}(111)$ are also shown. It is seen that $\mathrm{d}_{x z}\left(\mathrm{~d}_{y z}\right)$ and $\mathrm{d}_{z^{2}}$ states shift towards and away from $E_{\mathrm{F}}$, respectively when atomic $O$ undergoes weak hybridization with the surface-state, which is consistent with the above ARPES observations. In addition, the $\mathrm{d}_{z^{2}}$ orbital does not form significant bonding with the $\mathrm{s}$ or $\mathrm{p}$ orbital of $\mathrm{O}$, which indicates that the $\mathrm{S}_{\mathrm{K}}$ state may not be involved in any chemical bonding with atomic $\mathrm{O}$ on the $\mathrm{Pt}$ surface. The opposite direction of band shifts in $\mathrm{d}_{x z}\left(\mathrm{~d}_{y z}\right)$ and $\mathrm{d}_{z^{2}}$ results in a wider valence-band width.

\section{Conclusion}

In conclusions, we revealed the fundamental bonding interaction of chemisorbed atomic $O$ on the Pt(111) surface using ARPES measurements and DFT calculations. The electronic band structure of the Pt(111) surface showed a multitude of surface-states near the Fermi level. Among these surface-states, the one that has $\mathrm{d}_{x z}\left(\mathrm{~d}_{y z}\right)$ character around the $\mathrm{M}$ symmetry point participates in the bonding interaction with the $\mathrm{s}$ or $\mathrm{p}$ orbital of the $\mathrm{O}$ atom. Weak hybridization resulted from the chemical adsorption process and was attributed to minimal charge transfer between the Pt surface atoms and the $\mathrm{O}$ adsorbate atoms.

It might be worth mentioning about the relationship between our finding and the d-band model in which adsorbate-TM surface interactions are largely dependent on the center of d-bands. Since we focused only on the Pt surface, we could not draw the relationship between the present study and d-band center model. Instead, we believe that this work contributes to understanding the details of the d-band model as we verified which component in the surface DOS is related to the bonding with oxygen.

It is widely accepted that high efficiency in the catalytic reaction is achieved when the binding between the adsorbate and the surface is neither too strong nor too weak. The present work supports this picture by showing that the weak hybridization between $\mathrm{O}$ and the surface state of Pt leads to smaller adsorption energy of atomic oxygen. ${ }^{31}$ A better understanding of the microscopic picture of surface oxidation of $\mathrm{Pt}$ will provide significant groundwork for Pt-based catalyst systems.

\section{Acknowledgements}

This was supported by the Institute of Basic Science (IBS) (EM1203) and National Research Foundation of Korea (NRF) grant funded by the Korea government (MEST) (No. 2009-0068720, 2012R1A1A2001745, Priority Research Centers Program: No. 2009-0093818, and No. 2010-0020416). Also, this work and ALS were supported by the U.S. Department of Energy, Office of Basic Sciences under Contract No. DE-AC02-05CH11231. S.H.J. and S.W. were supported by the Center for Multiscale Energy System. The first-principles computations were carried 
out at KISTI (KSC-2012-C3-08). A.L.W. acknowledges support from the Max Planck Society. This paper was supported by GIST College's 2013 GUP Research Fund.

\section{Notes and references}

1 B. L. M. Hendriksen and J. W. M. Frenken, Phys. Rev. Lett., 2002, 89, 046101.

2 X.-Q. Gong, Z.-P. Liu, R. Raval and P. Hu, J. Am. Chem. Soc., 2004, 126, 8-9.

3 M. D. Ackermann, T. M. Pedersen, B. L. M. Hendriksen, O. Robach, S. C. Bobaru, I. Popa, C. Quiros, H. Kim, B. Hammer, S. Ferrer and J. W. M. Frenken, Phys. Rev. Lett., 2005, 95, 255505.

4 R. B. Getman, W. F. Schneider, A. D. Smeltz, W. N. Delgass and F. H. Ribeiro, Phys. Rev. Lett., 2009, 102, 076101.

5 E. M. Larsson, C. Langhammer, I. Zoric and B. Kasemo, Science, 2009, 326, 1091-1094.

6 A. D. Smeltz, R. B. Getman, W. F. Schneider and F. H. Ribeiro, Catal. Today, 2004, 136, 84-92.

7 D. Strmcnik, K. Kodama, D. van der Vliet, J. Greeley, V. R. Stamenkovic and N. M. Markovic, Nat. Chem., 2009, 1, 466-472.

8 L. Qi, X. Qian and J. Li, Phys. Rev. Lett., 2008, 101, 146101.

9 B. Hammer, L. B. Hansen and J. K. Norskov, Phys. Rev. B: Condens. Matter Mater. Phys., 1999, 59, 7413-7421.

10 W. A. Brown, R. Kose and D. A. King, Chem. Rev., 1998, 98, 797-831.

11 J. Wintterlin, R. Schuster and G. Ertl, Phys. Rev. Lett., 1996, 77, 123-126.

12 D. C. Ford, Y. Xu and M. Mavrikakis, Surf. Sci., 2005, 587, 159-174.

13 A. C. Luntz, J. Grimblot and D. E. Fowler, Phys. Rev. B: Condens. Matter Mater. Phys., 1989, 39, 12903-12906.
14 C. Puglia, A. Nilsson, B. Hernniis, O. Karis, P. Bennich and N. Martensson, Surf. Sci., 1995, 342, 119-133.

15 P. D. Nolan, B. R. Lutz, P. L. Tanaka, J. E. Davis and C. B. Mullins, J. Chem. Phys., 1999, 111, 3696-3704.

16 X.-G. Wang and G. B. Fisher, Phys. Rev. Lett., 2007, 99, 066101. 17 B. C. Stipe, M. A. Rezaei and W. Ho, J. Chem. Phys., 1997, 107, 6443-6447.

18 A. Eichler and J. Hafner, Phys. Rev. Lett., 1997, 79, 4481-4484. 19 B. Hammer and J. K. Norskov, Nature, 1995, 376, 238-240.

20 P. J. Feibelman, S. Esch and T. Michely, Phys. Rev. Lett., 1996, 77, 2257-2260.

21 J. F. Weaver, J.-J. Chen and A. L. Gerrard, Surf. Sci., 2005, 592, 83-103.

22 Z. Gu and P. B. Balbuena, J. Phys. Chem. C, 1997, 11, 9877-9883.

23 Y. S. Kim, A. Bostwick, E. Rotenberg, P. N. Ross, S. C. Hong and B. S. Mun, J. Chem. Phys., 2010, 133, 034501.

24 A. Eichler, F. Mittendorfer and J. Hafner, Phys. Rev. B: Condens. Matter Mater. Phys., 2000, 62, 4744-4755.

25 C. Enderlein, Y. S. Kim, A. Bostwick, E. Rotenberg and K. Horn, New J. Phys., 2010, 12, 033014.

26 H. Huang and J. Hermanson, Phys. Rev. B: Condens. Matter Mater. Phys., 1985, 32, 6312-6318.

27 P. E. Blöchl, Phys. Rev. B: Condens. Matter Mater. Phys., 1994, 50, 17953-17979.

28 J. P. Perdew, K. Burke and M. Ernzerhof, Phys. Rev. Lett., 1996, 77, 3865-3868.

29 G. Kresse and J. Hafner, Phys. Rev. B: Condens. Matter Mater. Phys., 1993, 47, 558-561.

30 We compared two distinct configurations, i.e., $1 \times 1 \times 7$ and $2 \times 2 \times 7$ Pt-supercell, and we obtained a similar tendency in the PDOS of surface Pt due to the chemical bonding with atomic oxygen.

31 V. Stamenkovic, et al., Angew. Chem., Int. Ed., 2006, 45, 2897-2901. 